\title{
Small Intestinal Perforation, CTCAE 5.0
}

National Cancer Institute

\section{Source}

National Cancer Institute. Small Intestinal Perforation, CT CAE 5.0. NCI Thesaurus. Code C146635.

A disorder characterized by a rupture in the small intestine wall. 